\title{
Archipel
}

ARCHIPEL Études interdisciplinaires sur le monde insulindien

$101 \mid 2021$

Varia

\section{In Memoriam Jacques Dumarçay (1926-2020)}

\section{Hélène Njoto}

\section{OpenEdition}

\section{Journals}

Édition électronique

URL : https://journals.openedition.org/archipel/2423

DOI : 10.4000/archipel.2423

ISSN : 2104-3655

\section{Éditeur}

Association Archipel

\section{Édition imprimée}

Date de publication : 30 juin 2021

Pagination : $3-10$

ISBN : 978-2-910513-85-6

ISSN : 0044-8613

\section{Référence électronique}

Hélène Njoto, «In Memoriam Jacques Dumarçay (1926-2020) », Archipel [En ligne], 101 | 2021, mis en ligne le 12 juin 2021, consulté le 16 juillet 2021. URL : http://journals.openedition.org/archipel/2423 : DOI : https://doi.org/10.4000/archipel.2423 


\section{In Memoriam Jacques Dumarçay (1926-2020)}

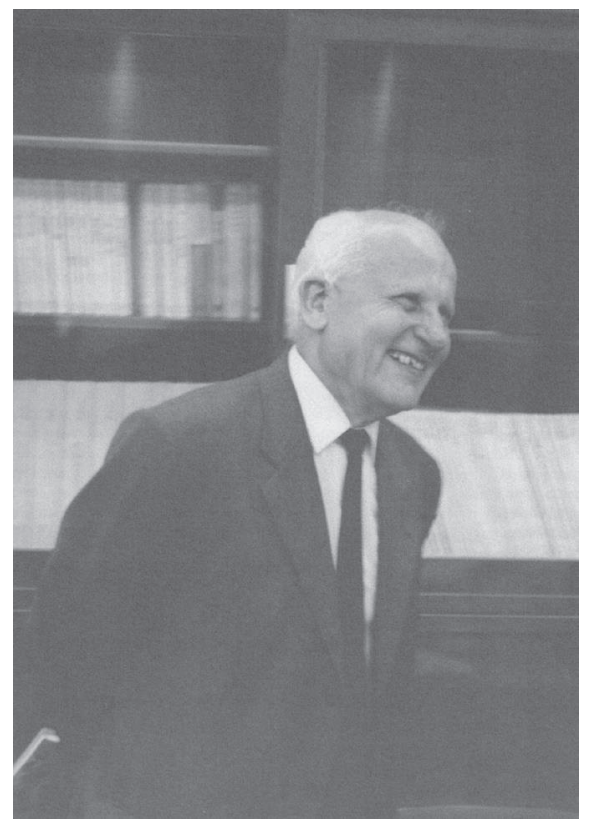

Jacques Dumarçay à Tokyo en 1997 (d'après H. Chambert-Loir et B. Dagens (éds.), Anamorphoses, 2006). 
Jacques Dumarçay s'est éteint le 22 novembre 2020 à l'âge de 94 ans à SaintRémy-lès-Chevreuse où il vivait avec son épouse Jacqueline sa collaboratrice dans de nombreux projets. La revue Archipel a voulu rendre hommage à un collègue, ami pour les uns, maître bienveillant et jovial pour la génération suivante. Il n'est pas rare d'entendre, qui, sur le terrain, non sans fierté, a tenu son mètre. Ces amitiés et les nombreuses collaborations qu'il entretint durant un peu plus de quarante années de carrière en Asie lui valurent de recevoir un volume d'hommage sous le titre Anamorphoses (2006)ํㅗㄴ. Mieux connu du public français pour ses travaux sur le Cambodge, notamment pour la restauration d'Angkor, Jacques Dumarçay a laissé un héritage tout aussi important dans les domaines de la conservation et de la connaissance du patrimoine architectural de l'Insulinde et de Java en particulier. Il fut en effet l'un des premiers, avec Denys Lombard, à introduire l'histoire de l'architecture et des jardins dans les études insulindiennes françaises, notamment à travers douze précieuses contributions publiées dans Archipel de 1974 à 2003 (voir liste infra).

C'est au milieu des années 1960 que Jacques Dumarçay s'engagea dans les études sud-est asiatiques, après avoir œuvré dix ans en Asie Centrale et au Pakistan auprès de la Délégation archéologique française en Afghanistan (DAFA). Le Cambodge fut le premier pays où il fut délégué, par Philippe Stern (1895-1979), alors directeur du musée Guimet et secrétaire général de la Mission archéologique des Indes, pour assister l'archéologue Bernard-Philippe Groslier (1926-1986) à Siem Reap, à la Conservation des monuments d'Angkor. Nommé chercheur à l'École française d'Extrême-Orient dès 1964, Jacques Dumarçay passa son diplôme d'architecte avant de soutenir un mémoire sur le Bayon (EHESS) ainsi qu' une thèse sur la charpenterie et les tuiles khmères (1971). Ces projets furent interrompus pendant deux ans par un séjour en Inde, où l'attendaient de nouvelles collaborations avec Bruno Dagens et Françoise L'Hernault (19371999). Il fut chargé de l'étude du temple du rivage de Mahabalipuram, et apportait ses conseils à la restauration indienne du temple de Darasuram. Enfin, il fut mandaté à Java en 1973 pour participer à la restauration du Borobudur où l'avait précédé quarante ans plus tôt, son compatriote et confrère Henri Marchal (1876-1970) pour observer les techniques de l'anastylose, pratiquée par l'archéologue néerlandais Th. Van Erp (1874-1958) et son équipe.

Ce séjour javanais d'une quinzaine d'années fut un moment prolifique de sa carrière, puisqu'il y produisit 11 des 35 ouvrages qu'il publia tout au long de sa carrière (la majorité publiée dans la série des Mémoires archéologiques de l'EFEO), ainsi que 26 articles ou contributions diverses. Il y a d'abord été

1. Bruno Dagens et Henri Chambert-Loir (éds.), Anamorphoses : hommage à Jacques Dumarçay. Paris : Les Indes savantes, 2006. Pour une note nécrologique exhaustive et une bibliographie complète se référer à l'article de Bruno Dagens, dans le prochain Bulletin de l'École française d'Extrême-Orient, n¹07/2021. 
détaché auprès de l'UNESCO pour œuvrer avec C. Voûte et le Professeur Soekmono (1922-1997), du Centre national de recherches archéologiques, au projet de restauration du Borobudur. Ce sont ses collaborations étroites avec les archéologues indonésiens qui lui permettront de mettre au point, en 1976, sous l'égide de Mme Satyawati Suleiman (1920-1988), directrice du Centre national de recherches archéologiques et de Jean Filliozat (19061982), directeur de l'EFEO, le contrat de coopération, toujours en vigueur, entre l'EFEO et ce partenaire indonésien.

D'abord installé avec son épouse Jacqueline, leur fille Anne et leur fils François dans une « belle maison avec vue sur le mont Sumbing » à Magelang, près du Borobudur, ils déménagèrent quelques années plus tard au cœur de Yogyakarta, dans une maison de kampung (village). Les premières années de sa carrière à Java furent consacrées à la publication de travaux antérieurs : sa thèse sur les charpentes et tuiles khmères (1973), ainsi que deux études sur ses recherches en Inde (1975), puis à son Histoire architecturale du Borobudur (1977), somme de ses relevés et analyses sur le temple. Simultanément aux travaux sur le Borobudur, il s'engagea dans un nouveau projet d'histoire architecturale du Candi Sewu, à la restauration duquel il collabora par la suite avec le Service du Patrimoine (Direktorat Perlindungan dan Pembinaan Peninggalan Sejarah Purbakala), jusqu'en 1987. L'étude des temples et la question de l'évolution des techniques tiennent une place de premier plan dans l'œuvre de Jacques Dumarçay. Il est connu pour avoir expérimenté notamment une méthode de datation des techniques de construction par association à des monuments datés grâce à l'épigraphie ou la statuaire.

Parallèlement à ses publications et à ses grandes collaborations à JavaCentre, il se lança également dans des recherches sur le début de la période moderne alors encore peu documenté. Installé à Yogyakarta, la dernière capitale royale javanaise fondée au XVIII ${ }^{\mathrm{e}}$ siècle, il en profita pour mener une nouvelle série d'études plus courtes, mais non moins utiles sur les mosquées, les palais et jardins palatins. Comme il l'avait déjà expérimenté au Cambodge en particulier, sa démarche diachronique et comparatiste consistait à mettre en regard les bâtiments tardifs pour comprendre l'architecture figurée dans les reliefs de la période indianisée, ceux du Borobudur et de Prambanan plus particulièrement. Cette méthode encore rarement appliquée à Java, était aussi le fruit d'une tradition française, celle de ses maîtres à l'EFEO, comme Henri Parmentier (1871-1949), qui l'avait précédé avec un article sur « L'architecture interprétée des bas-reliefs de Java » (BEFEO VII, 1907). L'Histoire architecturale de Java (1993) de Jacques Dumarçay, qui traite pourtant essentiellement des VIII ${ }^{e}-X I V^{e}$ siècles, inclut également une étude sur le $\mathrm{XV}^{\mathrm{e}}$ siècle transitoire et deux autres études, menées avec des collègues indonésiens, sur les mosquées et la période coloniale, un travail qu'il supervisa avec cette même volonté de mettre en regard architectures anciennes et modernes. 
Or, en Insulinde, cette démarche comparatiste était tout autant un prétexte pour étudier le transfert des anciennes formes architecturales aux périodes moderne et contemporaine. Contrairement au Cambodge et à l'Inde, les pagodes, monastères et temples en bois y avaient disparu en réponse à l'islamisation, mais certains types architecturaux furent transformés et adaptés pour le nouveau culte musulman. Fort de son expertise régionale sur la charpenterie, il voyait dans les mosquées, les pavillons royaux et domestiques (pendapa) avec leurs toits superposés, leurs soubassements et leurs charpentes rayonnantes des héritiers directs des anciens temples et palais figurés. Sa fascination pour les mosquées dépassait d'ailleurs le monde javanais, puisqu'il publia deux autres articles dans Archipel sur les mosquées de type malais : le Langgar tinggi du quartier des musulmans indiens (Pekojan) à Jakarta (Archipel, 1985, vol. 30) et la mosquée de Kampung Laut en Malaisie (Archipel, 1992, vol. 44).

Tout aussi curieux des palais et jardins royaux que des mosquées, il publia sa première «Étude architecturale », titre d'une série d'articles, sur le jardin royal de Yogyakarta, le Taman Sari (1978). Ce premier moment de l'histoire coloniale javanaise, qui n'avait pratiquement pas retenu l'attention des archéologues et historiens néerlandais de l'architecture, avait suscité pour Jacques Dumarçay un grand intérêt. Outre le palais de Yogyakarta, c'est aussi celui de Plered, l'une des premières capitales royales de la dynastie Mataram au $\mathrm{XVII}^{\mathrm{e}}$ siècle, qui retint son attention au point qu'il y effectua quelques relevés (Archipel 1989, vol. 37), comme il le fit pour d'autres résidences princières plus tardives tombées en ruine dans la banlieue de Yogyakarta (Rejawinangun, Gua Seluman, etc.). Ces travaux devaient rejoindre d'autres études sur les palais d'Asie du Sud-Est dont il tira une monographie publiée en anglais (1991). C'est également dans les années 1990 qu'il collabora à l'étude d'un autre sultanat javanais, Banten, situé à l'ouest de l'île (1990).

Dans son étude sur le Taman Sari au XVIII e siècle, davantage que le symbolisme du jardin, ce fut l'usage intégral de la technique de la maçonnerie à mortier qui l'intéressait. Il y vit un moment singulier de créativité à Java, où les maîtres d'œuvre javanais relevèrent un défi de taille, celui de raviver d'anciennes formes architecturales et éléments de décor en bois dans des matériaux nouveaux. L'adoption des nouvelles techniques de construction, sur laquelle il publia une monographie sur le même sujet chez Brill (2005), le fascinait particulièrement. À Java et à Bali, l'adoption de la charpente rayonnante venue d'Inde du Sud permit d'agrandir les espaces intérieurs. La question des contraintes induites par de nouvelles circonstances économiques ou techniques, comme les changements de matériaux, est omniprésente dans l'œuvre de Jacques Dumarçay. On se souvient, notamment, du problème récurrent dans son œuvre de la « disparition des grands fûts », c'est-à-dire des arbres de haute futaie, qui a entraîné l'abandon des maisons à faîtière tendue, mais aussi de la charpente rayonnante au profit de la charpente triangulée. Dans certaines maisons toraja traditionnelles, cette contrainte matérielle n'aurait pas 
été anticipée et les anciens modèles continuèrent à être construits, mais avec quelques désavantages : les pignons élancés, qui reposaient désormais sur des poutres plus courtes, devaient être soutenus à l'extérieur par un long poteau.

Jacques Dumarçay se plaisait à déceler les irrégularités et curiosités architecturales. C'est un des aspects particulièrement attachants et souvent amusants de son travail. Il aimait identifier les failles, les défis techniques non relevés, les problèmes de raccord (dans les temples Pallava), les problèmes d'étanchéité aussi dans les bassins monumentaux à Angkor (Bayon), mais aussi neuf siècles plus tard à Java, dans les couloirs subaquatiques suintants, menant à l'île artificielle (Pulo Kenanga) du Taman Sari. C'est avec beaucoup d'affection pour les maîtres d'œuvre qu'il décrivait la façon dont, au Candi Gedong Songo par exemple, ils avaient voulu incruster le seuil dans le soubassement rendant le programme architectural intérieur tout à fait incohérent avec celui exposé sur la façade du temple. Au Cambodge, dans les tours sanctuaires de Prasat Kravanh (IX $-\mathrm{X}^{\mathrm{e}}$ siècles), ce sont les bases des pilastres sur les petites tours qui disparaissaient maladroitement de la vue du fait de la taille trop importante des corniches.

Ces effets, plus ou moins heureux, tiennent une place importante dans son travail. Les effets perspectifs, auxquels il consacra un ouvrage entier (1983) sont un procédé visuel dont Jacques Dumarçay était devenu expert. Exigeant des calculs complexes, l'effet permettait de grandir de façon artificielle un monument en réduisant proportionnellement les éléments en hauteur. Il montra comment il fut appliqué en Inde, à Java et au Cambodge, où le procédé fut abandonné bien que les formes architecturales en fussent gardées. Au-delà des effets visuels et des maîtres d'œuvre, c'est le regard d'une société et de ses hommes qui préoccupait J. Dumarçay. Lorsqu'il recommandait de « lire » dans l'œuvre achevée, c'est pour mieux comprendre les créateurs : les maîtres d'œuvre, mais aussi les maitres d'ouvrage (commanditaires). Il met en avant l'importance du sens de lecture d'un monument, guidé par les reliefs narratifs, mais aussi l'interruption de ce genre de « lecture suivie » au XIVe siècle à Java. Il insiste aussi sur l'intérêt du « spectacle» dans les jardins (2000) et du «parcours » à l'intérieur des maisons, partout où un sens de lecture précis était suggéré par l'architecte.

C'est ce qu'il s'efforça d'illustrer dans ses deux derniers ouvrages : L'architecture et ses modèles en Asie méridionale (1998) et Babel ruinée (1996). Ce "Petit traité du regard porté sur l'architecture en Asie méridionale », en dit autant sur l'œuvre achevée que sur l'esprit des bâtisseurs et l'« intelligence collective » des sociétés anciennes. Jacques Dumarçay leur consacra un ouvrage Le savoir des maîtres d'œuvres à Java (1986) et deux articles, dont un dans les Annales (1998) sur la permanence des modèles et la mémoire collective, où les bâtisseurs venaient puiser, telles des guêpes maçonnes, lorsqu'ils devaient construire des modèles éprouvés. Avant tout, Jacques Dumarçay a su rendre vie, grâce à une écriture rigoureuse et simple, aux maîtres d'œuvre du passé, sur lesquels on est par ailleurs si mal renseignés. 
Cette fascination pour le processus créateur ne se limitait pas à l'architecture. Il s'intéressa à d'autres formes artistiques, auxquelles il fit appel dans ses démonstrations où l'image tient d'ailleurs une place très importante. C'est cette approche multidisciplinaire qui fait aussi le charme de son travail. On le sait moins, Jacques Dumarçay est l'auteur de nombreuses (21) recensions bibliographiques, dont les sujets variés montrent l'étendue de sa curiosité. Les représentations sur divers supports, comme les lithographies ou encore les fixés sous verre, qu'il collectionnait et qu'il aimait redessiner au trait dans un style qui lui était propre, n'en sont que deux exemples. L'amour de la littérature l'accompagna également tout au long de sa carrière. Initié très tôt à cet art par son père agrégé de lettres et spécialiste notamment de Jean de La Fontaine, il avait développé un goût pour les narrations dans les reliefs sud-est asiatiques et les récits locaux. Il livra, par ailleurs, un compte-rendu savant sur des miniatures d'Imam Bakhsh Lahori (1991) inspirées des fables de La Fontaine. Trois ans plus tard, il rédigea Les sources orientales de Jean de La Fontaine à Java (1993), un livret illustré où il démontre comment les sculpteurs javanais puisèrent, en partie, aux mêmes sources que Jean de La Fontaine (Pancatantra, Jataka, etc.).

Si nous avons tenu à rendre hommage à de nombreux aspects moins connus de son travail en Insulinde, il reste que Jacques Dumarçay demeure avant tout pour le public français " le grand architecte au chevet d'Angkor », comme le titrait la demi-page du Monde qui lui a été consacrée le 10 décembre 2020. C'est en effet à Angkor, où sa carrière sud-est asiatique avait commencé, qu'il effectua ses dernières missions pour l'EFEO. Retraité depuis un an, on lui confia le projet de restauration de la Terrasse du Roi Lépreux (1993) puis du Baphuon (1995) qu'il avait dû abandonner vingt ans auparavant, au début de la guerre civile cambodgienne. Il contribua également à assurer la relève des travaux d'archéologie et de restauration au Cambodge en prenant sous sa direction en 1992 de jeunes chercheurs, devenus par la suite Directeurs des Études à l'EFEO, Christophe Pottier et Pascal Royère (1965-2014), qui poursuivirent ses travaux. Enfin, il faut rappeler que Jacques Dumarçay n'est pas uniquement connu du public français. Dès les années 1980, il publie avec Michael Smithies principalement de nombreux travaux de vulgarisation chez Brill, Oxford University Press ou encore chez Gramedia (13 ouvrages au total), lesquels sont devenus des références bibliographiques incontournables pour l'enseignement de l'histoire architecturale en Asie du Sud-Est. Il prodiguait conseils et explications avec un enthousiasme constant, et ceux qui le voulaient, jeunes chercheurs, étaient les bienvenus à St-Rémy-lès-Chevreuse, où ils étaient reçus avec chaleur. Ce que les jeunes archéologues et les historiens de l'architecture de cette "Asie méridionale » doivent à Jacques Dumarçay est inestimable, tant pour ses travaux que pour les pistes de réflexion qui abondent dans toute son œuvre.

Hélène Njoto

École française d'Extrême-Orient, Jakarta 


\section{Ouvrages et articles de Jacques Dumarçay auxquels il est fait référence dans le texte (les publications dans Archipel sont signalées par un astérisque)}

1973, Charpentes et tuiles khmères, Paris : EFEO (Mémoires archéologiques, 8).

1973, « Les charpentes rayonnantes sur plan barlong ou carré de l'Asie méridionale », BEFEO 60, p. 85-104.

1974*, «Les charpentes figurées de Prambanan », Archipel 7, p. 139-150. [trad. anglaise 1994 ci-dessous].

1975, Temples pallava construits. (avec Françoise L'Hernault), Paris : EFEO (Mémoires archéologiques, 9).

1977, Histoire architecturale du Borobodur (avec le Badan Pemugaran Candi Borobudur et François Grenade), Paris : EFEO (Mémoires archéologiques, 12).

1978, « Le Taman Sari (Étude architecturale) », BEFEO 65(2), p. 589-624.

1981, Candi Sewu et l'architecture bouddhique du Centre de Java. (avec Pascal Lordereau), Paris : EFEO (Mémoires archéologiques, 14) [voir ci-dessous traductions indonésienne et anglaise $1986 \& 2007]$.

1981, « La faîtière tendue (histoire d'une technique) », BEFEO 70, p. 231-252.

1982, « Notes d'architecture javanaise et khmère », BEFEO 82, p. 87-147.

1983, Les effets perspectifs de l'architecture de l'Asie méridionale, Paris : EFEO

(Mémoires archéologiques, 15).

1985*, «Le Langgar Tinggi de Pekojan, Jakarta » (avec Henri Chambert-Loir), Archipel 30, p. $47-56$.

1985*, « La charpenterie des mosquées javanaises », Archipel 30, p. 21-30.

1986, Le savoir des maîtres d'œuvre javanais aux XIII et XIV̋ siècles, Paris, EFEO (Mémoires archéologiques, 17).

1986*, «L'espace architectural indo-javanais », Archipel 31, p. 73-85.

1986, Les sources orientales de Jean de la Fontaine à Java, Yogyakarta : LIP (Alliance française).

1986, Candi Sewu dan Arsitektur Bangunan Agama Buda di Jawa Tengah (diterjemahkan oleh

Winarsih Arifin dan Henri Chambert-Loir), Jakarta : EFEO, Pusat Penelitian Arkeologi Nasional.

1989*, « Plered, capitale d'Amangkurat I ${ }^{\mathrm{er}} »$, Archipel 37, p. 189-198.

1990, The Sultanate of Banten (avec Claude Guillot \& Hasan M. Ambary), Jakarta : Gramedia. 1990, Borobudur : sanctuaire bouddhiste de Java, (avec Soekmono \& Johannes G. de Casparis) [traduit de l'anglais par Marialys Bertault], Paris : Arthaud.

1991, The palaces of South-East Asia : architecture and customs (translated and edited by Michael Smithies), Singapore : Oxford University Press.

1991, Jean de La Fontaine : Le songe d'un habitant du Mogol et autres fables illustrées par Imam Bakhsh Lahori, BEFEO 78, p. 346-347.

1992*, «La mosquée de Kampung Laut (Kelantan) : étude architecturale », Archipel 44, p. 115-122.

1994, "The Beamwork Illustrated at Prambanana", Indonesia, 1994 / Archipel No 57 (April), pp. 5-14.

1996, Babel ruinée : petit traité du regard porté sur l'architecture en Asie méridionale, Paris : Librairie Oriens. 
1998, L'architecture et ses modèles en Asie du Sud-Est, Paris : Librairie Oriens.

1998, «Intelligence collective et architecture en Asie du Sud-Est », Annales. Histoire, Sciences sociales 53(3), p. 505-535.

1998*, « Note sur le second état du Candi Lumbung et du Candi Bima », Archipel 56, p. 455-464.

2000, « Le spectacle du jardin dans l'Asie de l'Est et l'Asie du Sud-Est : contrastes et similitudes », Extrême Orient - Extrême Occident 22, p. 167-173.

2002*, « Le Candi Badut », Archipel 63, p. 7-14.

2003*, «Les techniques de construction à Java, du VIII ${ }^{e}$ au XIV siècle », Archipel 66, p. 13-28.

2001, Compte-rendus de : Marijke J. Klokke (ed.), Narrative sculpture and literary traditions in South and Southeast Asia, Leiden, Brill, 2000, Archipel 61, p. 186-188.

Nora A. Taylor (ed.), Studies in Southeast Asian Art, Essays in Honor of Stanley J. O'Connor, Ithaca, New York, 2000, Archipel 61, p. 188-192.

2003*, « Un lac autour du Borobudur ? », Archipel 65, p. 17-24.

2007, Candi Sewu dan Arsitektur Bangunan Agama Buddha di Jawa Tengah (traduit par Winarsih Arifin et Henri Chambert-Loir) / Candi Sewu and Buddhist Architecture of Central Java (traduit par John N. Miksic), Jakarta : EFEO, Kepustakaan Populer Gramedia.

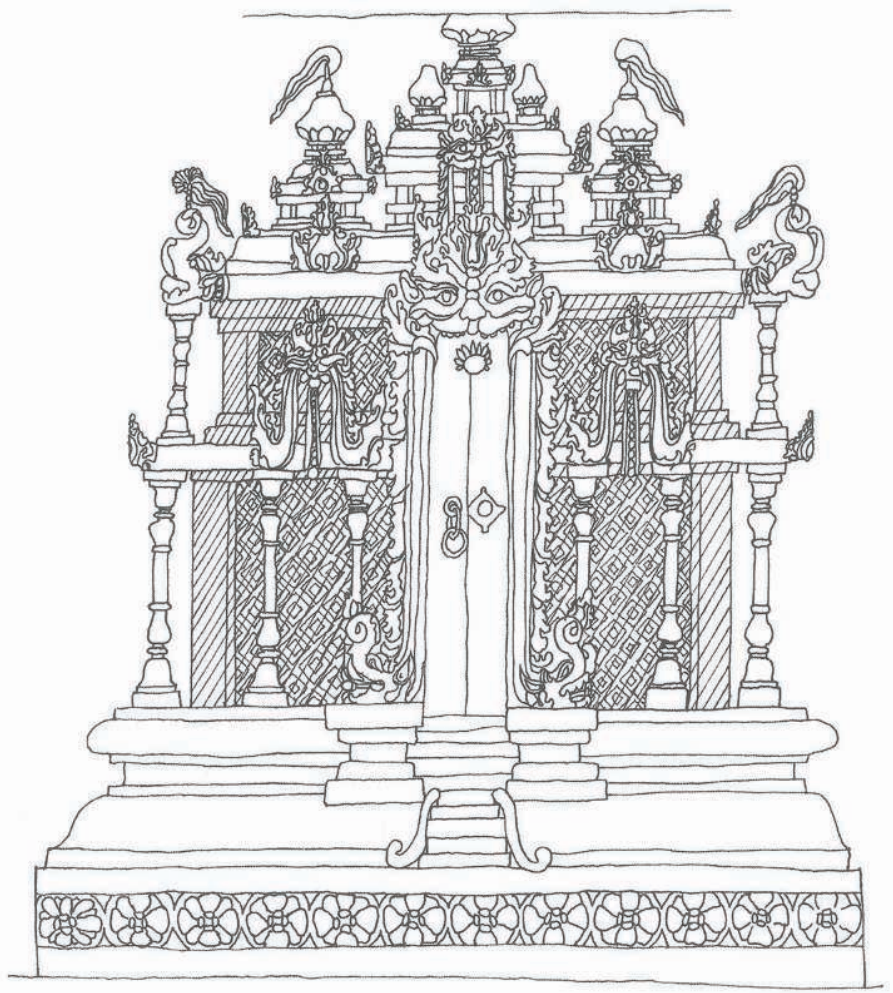

Dumarçay 2003, p. 16, fig. 2, Relief E/3/13 du Borobudur 